\title{
Exact mean first-passage time on the T-graph
}

\author{
E. Agliari ${ }^{1}$ \\ ${ }^{1}$ Dipartimento di Fisica, Università degli Studi di Parma, viale Usberti 7/A, 43100 Parma, Italy
}

(Dated: November 14, 2018)

\begin{abstract}
We consider a simple random walk on the T-fractal and we calculate the exact mean time $\tau^{g}$ to first reach the central node $i_{0}$. The mean is performed over the set of possible walks from a given origin and over the set of starting points uniformly distributed throughout the sites of the graph, except $i_{0}$. By means of analytic techniques based on decimation procedures, we find the explicit expression for $\tau^{g}$ as a function of the generation $g$ and of the volume $V$ of the underlying fractal. Our results agree with the asymptotic ones already known for diffusion on the T-fractal and, more generally, they are consistent with the standard laws describing diffusion on low-dimensional structures.
\end{abstract}

PACS numbers: 05.40.Fb

\section{INTRODUCTION}

Many problems in physics and chemistry are related to random walks on fractal structures [1, 2, 3]. The main reason is that such structures are able to mimic the inhomogeneity and scale-invariance typical of disordered materials. An important class of fractal structures is given by the so called exactly decimable fractals which include deterministic finitely ramified fractals such as the Sierpinski gasket and the T-graph. These structures, being amenable to renormalization procedures, allow exact analytic calculations [4, 5, 6].

In general, the lack of translational invariance implies significant corrections to the standard laws describing diffusion on regular lattices. Indeed, in the fields of reactiondiffusion and transport theory, a question of longstanding interest concerns the interplay between spatial extent and system dimensionality in affecting the reaction kinetics and the transport efficiency [7].

A fundamental quantity characterizing diffusion is the mean first-passage time (MFPT), i.e. the expected time for a random walker, starting with equal probability at any site $i \neq i_{0}$, to first reach a given site $i_{0}$. This problem was first set up by Montroll [8] in the case of regular structures and later extended to more complex substrates $[9,10,11,12,13,14,15]$. Notice that this definition of MFPT involves a double average: the first one is over all the walks from a given origin $i$, then you must average over a uniform distribution of initial sites, whose support is the whole set of graph sites, except $i_{0}$.

The mean first-passage time is also intrinsically related to a number of different problems [12]. In the context of reaction-diffusion processes it represents the mean time to react for a particle diffusing in the presence of an active site located in $i_{0}[8,16,17,18]$, which is sometimes referred to as target problem. Not only: the mean firstpassage time defined above also describes the asymptotic behaviour of the average time for two diffusive particles to first encounter [19].

The MFPT has been previously studied on different kinds of structures and several analytical results have been found. Most of them consists of scaling relations

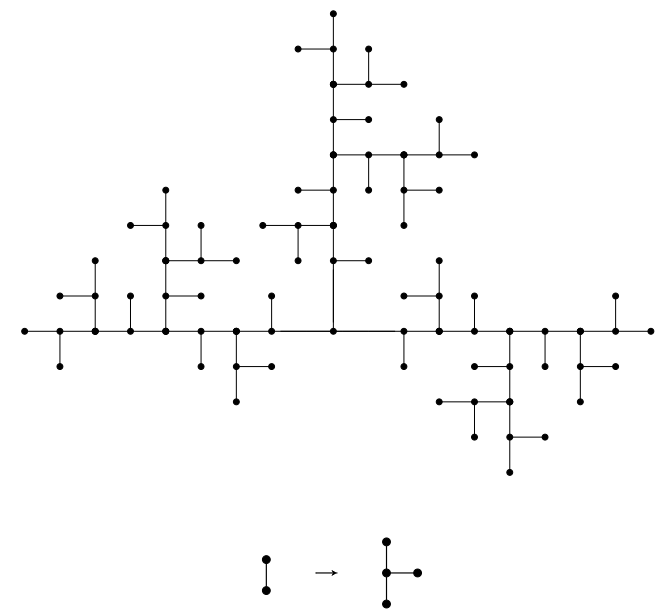

FIG. 1: T-fractal of generation $4: V=3^{4}+1$. The next generation is obtained by performing the operation illustrated in the bottom on each bond.

and asymptotic behaviours 9, 13, 14], while a very few exact results are known [11, 12]. Exact solutions on finite structures are especially longed for since they prove useful for a deeper comprehension of theoretical models and for checking approximate solutions or numerical simulations.

Here, we derive the exact mean first-passage time for a random walker on the T-fractal, following the decimation procedure recently introduced by Kozak et al. and applied to the Sierpinski gasket [11]. In particular, we assume a simple random walker (RW) in the presence of a perfect trap fixed at the central site and we calculate the mean-walk length before absorption. The closed-form expression we obtain for the latter, is akin to the one in [1] and consistent with known asymptotic results [9, 10, 19]. Though they are both deterministic fractals, the Sierpinski gasket and the T-fractal display significant differences: while the former models self-similar structures endowed with closed loops, the T-fractal is representative 
for tree-like structures and bundled structures in general [9, 10, 20, 21, 22, 23]. For this reason it is worth extending and comparing the related results.

The paper is organized as follows. In Sec. [II we describe the main features of the T-graph and we resume the analytic background underlying the analytic solution; in Sec. III we describe the decimation procedure applied and we obtain the exact formula for the mean first-passage time as a function of both the generation and the volume of the structure; finally Sec. IV includes conclusions and comments.

\section{THEORY}

\section{A. Exactly Decimable Fractals}

A generic graph $\mathcal{G}$ is mathematically specified by the pair $\{\Lambda, \Gamma\}$ consisting of a non-empty, countable set of points, $\Lambda$ joined pairwise by a set of links $\Gamma$. The cardinality of $\Lambda$ is given by $|\Lambda|=V$ representing the number of sites making up the graph, i.e. its volume. From an algebraic point of view, a graph $\mathcal{G}=\{\Lambda, \Gamma\}$ is completely described by its adjacency matrix $A$. Every entry of this off-diagonal, symmetric matrix corresponds to a pair of sites, and it equals one if and only if this couple is joined by a link, otherwise it is zero. The number of nearest-neighbours of the generic site $i$, referred to as coordination number, can be recovered as a sum of adjacency matrix elements: $z_{i}=\sum_{j \in \Lambda} A_{i j}$. These are used to build up the diagonal matrix $Z_{i j}=z_{i} \delta_{i j}$ [6].

A very special class of graphs is given by the so called exactly decimable fractals which are geometrically invariant under site decimation. In general, all deterministic, finitely ramified fractals are exactly decimable. The solution of both the random walk and harmonic oscillations problems can be obtained by standard renormalization group calculations based on real space decimation procedures [4, 5, 6]. The Sierpinski gasket, the T-graph (Fig. 1), the branched Koch curves are examples of exactly decimable fractals, which accounts for their popularity. Notice that such structures are characterized by strong restrictions on their topology which can give rise to properties far from holding for all fractals [20].

Here, we consider a T-fractal which is iteratively constructed by performing the operation illustrated in Fig. 1 on each link. The number of iterations is called the generation $g$ of the fractal. At the $g$-th generation the cardinality of the set of nodes $V(g)$, hereafter called volume, is given by: $V(g) \equiv\left|\Lambda_{g}\right|=3^{g}+1$. The T-fractal has fractal dimension $d_{f}=\frac{\log 3}{\log 2} \approx 1.584$ and spectral dimension $\tilde{d}=\frac{\log 9}{\log 6} \approx 1.226$. We recall that the former gives the dependence of the volume of the system on its linear size $L$ :

$$
V(g) \sim[L(g)]^{d_{f}}=\left(2^{g}\right)^{d_{f}},
$$

while the latter governs (among other phenomena) the
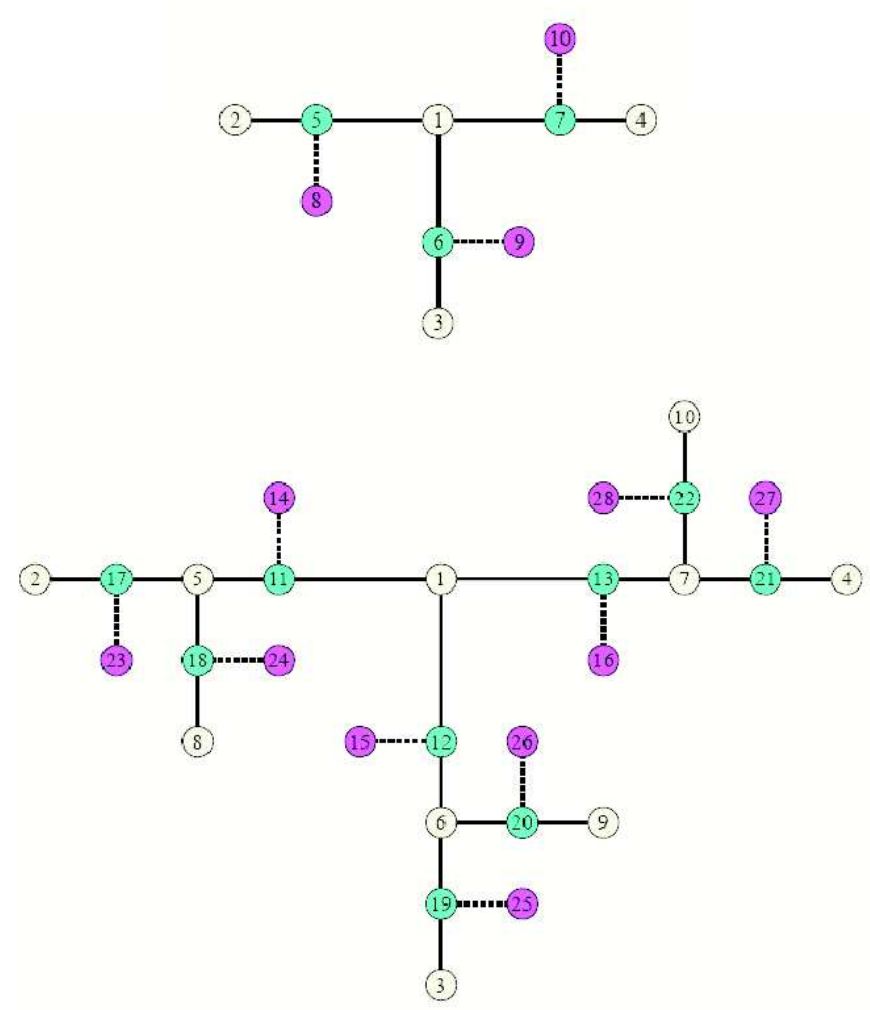

FIG. 2: (Color on line) T-graph of generation 2 (top) and 3 (bottom) with volume $V=3^{2}+1$ and $V=3^{3}+1$, respectively. Each site has been labelled according to the procedure described in the text. Dotted links belong to $\bar{\Gamma}^{g}$ and colored sites to $\bar{\Lambda}^{g}$; more precisely, pink sites are in $\bar{\Lambda}_{\text {ext }}^{g}$ and blue sites in $\bar{\Lambda}_{i n t}^{g}$

long-time properties of diffusion on the graph. Indeed, if we consider a random walker starting from a given site $i$ of the graph, the probability $P_{i i}(t)$ of returning back to the starting point at time $t$, at long times, follows the law

$$
P_{i i}(t) \sim t^{-\tilde{d} / 2}
$$

When $\tilde{d}<2$ the random walker is said to perform a "compact exploration" of the space [24] since the fractal dimension of its trajectory is greater than the dimension $d_{f}$ of the substrate.

It is worth underlining that the T-fractal is irregular, that is the coordination number is site dependent. We can distinguish among "internal site" with coordination number $z=3$ and "external site" with $z=1$. We call $\Lambda_{\text {int }}$ and $\Lambda_{\text {ext }}$ the set of internal and external sites respectively. Obviously $\Lambda^{g} \equiv \Lambda_{\text {int }}^{g} \cup \Lambda_{\text {ext }}^{g}$.

Figure 2 shows generations $g=2,3$ and the labelling method adopted, which will be useful in the next section. In each new generation, we label only the new sites while old sites keep their own labels. Hence, at gen- 
eration $g+1$ we name new sites progressively, starting from $V(g)+1$. The first sites to be labelled are the innermost, the last ones are those farthest from the central site $i_{0}=1$. At each generation we can distinguish sets of equivalent sites which are labelled anti-clock wise. Due to the symmetry of the T-graph, the cardinality of such sets is always a multiple of 3 ; for example we have $\{2,3,4,8,9,10\}_{g=2},\{14,15,16\}_{g=3}$.

Before proceeding further, let us resume some facts concerning unbiased diffusion on a T-fractal of generation $g$ represented by the adjacency matrix $A_{g}$ (henceforth we will omit the subscript $g$ ). We consider a simple RW, starting, at $t=0$, from site $i$; at each time step (taken to be unity) the particle jumps with equal probability to any of its nearest-neighbour sites. Being $P_{j i}(t)$ the probability of going from $i$ to $j$ in $t$ steps, the following Master equation holds:

$$
P_{j i}(t+1)=\sum_{k=1}^{V}\left(Z^{-1} A\right)_{j k} P_{k i}(t),
$$

which states that at each time step, the jumping probability from an internal site is $\frac{1}{3}$. From the previous equation it follows that $P_{j i}(t)=\left[\left(Z^{-1} A\right)^{t}\right]_{j i}$. It is also easy to verify that the Markov chain representing such a random walk is ergodic and the particle will visit all sites with probability 1 , independently of the origin $i$. Consequently, the walker will reach any site with probability 1 , in a time possibly diverging when $g \rightarrow \infty$.

\section{B. Mean Time to Absorption}

Let us consider a perfectly absorbing trap, fixed on the central site (labelled with index $i_{0}=1$ ) of the T-graph. Our aim is to obtain, through a decimation procedure, an exact, closed-form solution for the average time to absorption, where the average is meant both over all the possible walks starting from the same origin $i$ and over all sites $i \neq i_{0}$ taken as origin of the walk.

The special choice we made for the trap location makes the decimation procedure easier to be applied as we can identify the site $i_{0}$ since the first generation.

We now introduce $\tau_{i, q}^{g}$ to be the $q$-th $(q=0,1, \ldots)$ moment of the trapping time for a walk starting from $i$ on the $g$-th generation of the graph. Obviously, regardless of $g, \tau_{0, q}^{g}=0$ and $\tau_{i, 0}^{g}=1$. The latter is the zero-th moment of the distribution of the time to absorption given $i$ as origin and it is unitary because the walker will be trapped with probability 1 , whatever its origin.

The starting point for our analytic treatment is the discrete differential equation introduced in [11]:

$$
-\sum_{j=2}^{V(g)} \Delta_{i j} \tau_{j, q+1}^{g}=(q+1) \tau_{i, q}^{g},
$$

where $\Delta=A Z^{-1}-\mathrm{I}$ is a normalized version of the discrete Laplacian whose first row and column (correspond- ing to the trap site) have been removed. We recall that $\Delta$ is a nonsingular matrix and each row has sum zero, apart from those corresponding to sites $V(g-1)+1, V(g-1)+2$ and $V(g-1)+3$, i.e. the three nearest-neighbours of the trap, for which the sum is $-\frac{1}{3}$. As discussed in 7], Eq. 2 can be generalized to the case of two or more particles simultaneously diffusing.

In the following, we just focus on the set of first moments $\tau_{j, 1}^{g}$, for which Eq. (2) simplifies into

$$
-\sum_{j} \Delta_{i j} \tau_{j, 1}^{g}=\tau_{i, 0}^{g}=1 .
$$

Henceforth we can drop the index corresponding to $q$ without ambiguity:

$$
-\sum_{j} \Delta_{i j} \tau_{j}^{g}=1
$$

Now, we implement the average over the starting site $i$, chosen according to a uniform distribution in $\Lambda^{g} \backslash\left\{i_{0}\right\}$ :

$$
\tau^{g}=\frac{1}{V(g)-1} \sum_{i=2}^{V(g)} \tau_{i}^{g}=\frac{1}{V(g)-1} \sum_{i=2}^{V(g)} \sum_{j=2}^{V(g)}\left(-\Delta^{-1}\right)_{i j} .
$$

In the next Section we derive some recurrence relations which allow to simplify the previous equation.

Equation 3 can be very easily interpreted if we look at the random walk as a Markov chain. Indeed, $-\Delta^{-1}$ is just the fundamental matrix for the process, whose entry $i, j$ represents, by definition, the expected number of times that the process is in the transient state $j$, being started in the transient state $i$.

Finally, notice that, due to the symmetry and the absence of loops characterizing the structure under consideration, the mean time to absorption found in this case just corresponds to the mean time to reach either site 2, 3, 4 on a T-fractal of generation $g-1$. Otherwise stated, if we call $\pi^{g}$ the mean time to first reach site 2 (or, analogously, 3,4 ), then $\pi^{g}=\tau^{g+1}$.

\section{DECIMATION PROCEDURE}

The number of terms to sum up in Eq. 3 grows exponentially with $g$, hence, a direct calculation of $\tau_{i}^{g}$ and $\tau^{g}$ can be accomplished straightforwardly only for the very first generations (see Tab. I and (II). Such data allow to get some recurrence relations useful for the derivation of the final formula. First of all, notice that for a given site $i$, we have $\tau_{i}^{g+1}=6 \tau_{i}^{g}$ : in each generation the chemical distance from $i$ to the trap doubles while the mean time to first reach the trap increases by a factor 6 . This exact scaling follows from the symmetry and decimability of the graph and it is consistent with the random walk dimension on the T-fractal: $d_{w}=\frac{2 d_{f}}{\tilde{d}}=\frac{\log 6}{\log 2}$ [1].

Furthermore, at each generation $g$ we insert on the existing fractal some new links and some new vertices. We 
TABLE I: Mean time to absorption $\tau_{i}^{g}$ for a random walker starting from a given site $i$. For these values the average is only performed over all possible random walks sharing the same origin. Due to the symmetry of the T-fractal we can distinguish sets of equivalent sites such that, if taken as origin of the walk, they provide the same mean time $\tau_{i}^{g}$. Notice that, for the farthest sites from the trap, having chemical distance $2^{g-1}$, the average absorption time is $6^{g-1}$, consistently with the random walk dimension $d_{w}$.

\begin{tabular}{ccccccccccc}
\hline \hline$g \backslash i(2,3,4)$ & $(5,6,7)$ & $(8,9,10)$ & $(11,12,13)$ & $(14,15,16)$ & $(17,18,19,20,21,22)$ & $(23,24,25,26,27,28)$ & $(29,30,31)(32,33,34)$ \\
\hline 1 & 1 & - & - & - & - & - & - & - \\
2 & 6 & 5 & 6 & - & - & - & - & - \\
3 & 36 & 30 & 36 & 17 & 18 & 35 & - & - \\
4 & 216 & 180 & 216 & 102 & 108 & 210 & 216 & 53 \\
\hline \hline
\end{tabular}

call such sets $\bar{\Lambda}^{g}=\Lambda^{g} \backslash \Lambda^{g-1}$ and $\bar{\Gamma}^{g}=\Gamma^{g} \backslash \Gamma^{g-1}$, respectively. It is easy to see that $\left|\bar{\Lambda}^{g}\right|=V(g)-V(g-1)=$ $2 \cdot 3^{g-1}$ and $2 \cdot\left|\bar{\Gamma}^{g}\right|=\left|\bar{\Lambda}^{g}\right|$. Moreover, for each new link added we have a new couple of connected vertices $j_{\text {ext }}$ and $j_{i n t}$, belonging to $\bar{\Lambda}_{\text {ext }}^{g}$ and $\bar{\Lambda}_{\text {int }}^{g}$, whose coordination numbers are $z_{j_{e x t}}=1$ and $z_{j_{i n t}}=3$, respectively and:

$$
\begin{gathered}
\bar{\Lambda}^{g}=\bar{\Lambda}_{e x t}^{g} \cup \bar{\Lambda}_{i n t}^{g}, \\
\frac{1}{2} \bar{\Lambda}^{g}=\bar{\Lambda}_{e x t}^{g}=\bar{\Lambda}_{i n t}^{g} .
\end{gathered}
$$

For example, $\bar{\Lambda}_{\text {ext }}^{3}=\{14,15,16,23,24,25,26,27,28\}$, as shown in Fig. 2. Now, it is easy to see that

$$
\tau_{i_{e x t}}^{g}=\tau_{i_{i n t}}^{g}+1
$$

since a RW starting from $i_{\text {ext }}$ is necessarily on $i_{\text {int }}$ at time $t=1$. These facts hold regardless of the generation $g$ and for any connected couple chosen from $\bar{\Lambda}^{g}$. Thus we can write

$$
\sum_{i=2}^{V(g)} \tau_{i}^{g}=6 \sum_{i \in \Lambda^{g-1}} \tau_{i}^{g-1}+\sum_{i \in \bar{\Lambda}^{g}} \tau_{i}^{g}
$$

In the last sum we can highlight the contribution from external and internal sites and, exploiting Eqs. 4 and 5 .

$$
\sum_{i \in \bar{\Lambda}^{g}} \tau_{i}^{g}=\sum_{i \in \bar{\Lambda}_{e x t}^{g}} \tau_{i}^{g}+\sum_{i \in \bar{\Lambda}_{i n t}^{g}} \tau_{i}^{g}=2 \sum_{i \in \bar{\Lambda}_{i n t}^{g}} \tau_{i}^{g}+\left|\bar{\Lambda}_{i n t}^{g}\right|
$$

Let us now focus on the sum appearing in the right-most side and estimate it in the case $g=3$ depicted in Fig. 2 . The mean time to absorption for a RW starting from site 5 can be expressed as:

$$
\begin{aligned}
\tau_{5}^{3} & =1+\left(P_{11,5} \tau_{11}^{3}+P_{17,5} \tau_{17}^{3}+P_{18,5} \tau_{18}^{3}\right)= \\
& =\frac{\left(1+\tau_{11}^{3}\right)+\left(1+\tau_{17}^{3}\right)+\left(1+\tau_{18}^{3}\right)}{3}= \\
& =\frac{\tau_{14}^{3}+\tau_{23}^{3}+\tau_{24}^{3}}{3}
\end{aligned}
$$

where $P_{k i}$ represents the transition probability from state $i$ to state $k$. Hence, $\tau_{5}^{3}$ is just the average of the absorption times from 11,17,18, which mirrors the barycentric position of 5 with respect to the latters. Also notice that site 5 belongs to $\bar{\Lambda}_{i n t}^{g-1}$. Since these facts hold for any analogous subtree of generation 2 , we are allowed to write:

$$
\sum_{i \in \bar{\Lambda}_{e x t}^{g}} \tau_{i}^{g}=3 \cdot 6 \sum_{i \in \bar{\Lambda}_{i n t}^{g-1}} \tau_{i}^{g-1}
$$

From Eqs. 7 and 9 .

$$
\sum_{i \in \bar{\Lambda}_{i n t}^{g}} \tau_{i}^{g}+3^{g-1}=18 \sum_{i \in \bar{\Lambda}_{i n t}^{g-1}} \tau_{i}^{g-1}
$$

and solving this recurrence relation we obtain:

$$
\sum_{i \in \bar{\Lambda}_{i n t}^{g}} \tau_{i}^{g}=\frac{3^{g-1}}{5}\left(1+4 \cdot 6^{g-1}\right) .
$$

By plugging the last expression into Eq. (6) we get

$$
\begin{aligned}
(V(g)-1) \tau^{g}= & 6(V(g-1)-1) \tau^{g-1}+ \\
& +\frac{2}{5} \cdot 3^{g-1}\left(1+4 \cdot 6^{g-1}\right)+3^{g-1}
\end{aligned}
$$

and, dividing by $V(g)-1=3^{g}$ :

$$
\tau^{g}=2 \tau^{g-1}+\frac{1}{15}\left(7+8 \cdot 6^{g-1}\right) .
$$

The last expression is, again, a recursive equation, whose solution provides the exact time to absorption:

$$
\tau^{g}=\frac{1}{15}\left(-7+5 \cdot 2^{g}+2 \cdot 6^{g}\right)
$$

A numerical check of this formula can be attained by comparing $\tau^{g}, 1 \leq g \leq 6$ with data obtained by direct calculation and reported in Tab. II the agreement is perfect.

It is also possible to obtain an expression for $\tau^{g}$ as a function of the volume $V(g)=3^{g}+1$. In fact, recalling the spectral dimension for the $\mathrm{T}$-graph, we can write $2^{g}=$ $(V(g)-1)^{2 / \tilde{d}-1}$, and

$$
\tau^{g}=\frac{1}{15}\left[5(V(g)-1)^{2 / \tilde{d}-1}+2(V(g)-1)^{2 / \tilde{d}}-7\right] .
$$


TABLE II: Mean first-time $\tau_{g}$ obtained by direct calculation from Eq. 3. since this implies a sum over a number of terms exponentially increasing with $g$, only small generations have been considered.

\begin{tabular}{lcc}
\hline \hline $\mathrm{g}$ & $\mathrm{V}(\mathrm{g})$ & $\tau^{g}$ \\
\hline 1 & 4 & 1 \\
2 & 10 & $51 / 9$ \\
3 & 28 & $837 / 27$ \\
4 & 82 & $14391 / 81$ \\
5 & 244 & $254421 / 243$ \\
6 & 730 & $4550175 / 729$ \\
\hline \hline
\end{tabular}

Notice that the last expression gives the exact, explicit dependence of $\tau^{g}$ on $V(g)$. In the asymptotic limit, as $V(g)$ diverges,

$$
\tau^{g} \rightarrow V(g)^{2 / \tilde{d}} .
$$

This result is consistent with the leading behaviour of $\tau^{g}$ on the T-fractal discussed in [9] and, more generally, to the leading behaviour of the trapping time on low dimensional $(\tilde{d}<2)$ structures. In fact, as already remarked, the MFPT represents the mean trapping time $\tau_{\text {trap }}$ for a diffusing particle in the presence of a fixed perfect trap (or, symmetrically, the trapping time for an immobile target in the presence of a diffusive trap). Being $P_{s}(t)$ the survival probability at the $t$-th step $P_{s}(t)$, i.e. the probability that the RW has not yet reached the trap site, then [1]:

$$
\tau_{\text {trap }}=\int_{0}^{\infty}-\frac{\partial P_{s}(t)}{\partial t} \cdot t d t
$$

The asymptotic expression for the survival probability is [25]:

$$
P_{s}(t)=\exp \left[-\frac{\phi(t)}{V}\right]
$$

with

$$
\phi(t) \sim\left\{\begin{array}{cc}
t^{\tilde{d} / 2}, & \tilde{d}<2 \\
\frac{t}{\log t}, & \tilde{d}=2 \\
t, & \tilde{d}>2
\end{array}\right.
$$

One therefore expects that, for low dimensional structures, $\tau_{\text {trap }} \sim V^{2 / \tilde{d}}$. Our result and the one in [11] establish this relationship rigorously.

As can be shown by scaling arguments, on long times, the factor $6^{g} \sim V(g)^{2 / \tilde{d}}$, which is the leading term in Eqs. 13, 14, is involved in all the dynamical properties of diffusion on the T-fractal. For example, the characteristic time in the exponential decay of the survival probability increases with the generation of the tree as $6^{g}[9]$.

Equation 14 is also consistent with a recent result obtained by Condamin et al. [15] who found the asymptotic (large $V$ ) expression for the average time $\tau_{r}$ taken by a RW on a generic scale-invariant structure to first reach a

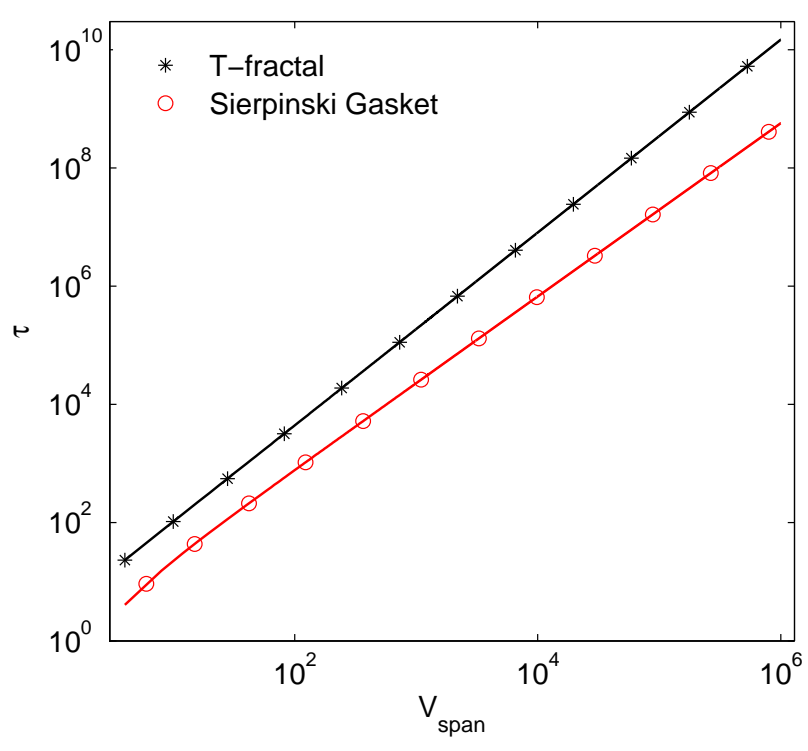

FIG. 3: (Color on line) Mean first-passage time for a simple random walker moving on a T-fractal (black stars) and on a Sierpinski gasket (red circles) as a function of the volume $V_{\text {span }}$ (the volume the RW can actually span before being trapped), according to exact analytic solutions.

trap distant $r$ from the starting point. In particular, for compact exploration $\left(d_{w} \geq d_{f}\right), \tau_{r} \sim V \cdot r^{d_{w}-d_{f}}$. Thus, if we fix the trap on a particular site $i_{0}$, we can obtain an estimate for the MFPT, by simply averaging over all possible distances $r$ from $i_{0}$. For the T-fractal considered here, $i_{0}=1$ and we can write:

$$
\tau^{g}=\frac{\sum_{r=0}^{L(g-1)} \tau_{r} n(r)}{V(g)}
$$

where $n(r)$ is the number of sites distant $r$ from $i_{0}$ and $L(g-1)$ is the largest distance from the central site. Under the above mentioned assumption of large volume, we can adopt a continuous picture and $n(r) \sim 3 \cdot r^{d_{f}-1}$; by integrating the previous expression, we get $\tau^{g} \sim V(g)^{2 / \tilde{d}}$, as expected.

Finally, in Fig. 3 we compare Eq. 11] with the analogous formula found for the Sierpinski gasket $\left(\tilde{d}=\frac{\log 9}{\log 5}\right)$ in [11]:

$\tau^{g}=\frac{2 V(g)-3}{V(g)-1}\left[\frac{(2 V(g)-3)^{\frac{2}{d}}}{6}+\frac{2(2 V(g)-3)^{\frac{2}{d}-1}}{5}-\frac{1}{6}\right]$.

In the asymptotic limit, $\tau^{g}$ diverges faster for the $\mathrm{T}$ fractal. This can be trivially drawn algebraically while, from a topological point of view, it evidences the role of loops in reducing the average distance between two random sites, making the diffusive particles survive shorter. 


\section{CONCLUSIONS}

In this work we study the mean first-passage time $\tau^{g}$ for a random walker on a T-fractal. The latter, being exactly decimable, allows the use of the powerful technique of exact renormalization. We find an exact, closed-form solution for $\tau^{g}$ as a function of either the generation $g$ and the volume $V(g)$. The leading term of $\tau^{g}$ is consistent with known asymptotic results. It should be underlined that an exact solution on a finite system is generally very useful in order to understand more quantitatively the asymptotic limit.

Our findings are interesting also in the light of a recent result concerning the survival probability for the trapping problem $A+B \rightarrow B$, with both species, $A$ and $B$, diffusing [19]. There, it was shown that on low-dimensional structures $(d<2)$ the survival probability for an A particle asymptotically does not depend on its diffusivity constant $D_{A}$. Otherwise stated, at long times, the target problem and the trapping problem provides the same results. Hence, exact results concerning the target problem, also provide the correct asymptotic behaviour for the trapping problem with diffusive traps.

The author is grateful to D. Cassi and R. Burioni for useful discussions and comments.
[1] D. ben-Avraham and S. Havlin, Diffusion and Reactions in Fractals and Disordered Systems, Cambridge University Press, 2004.

[2] V. Balakrishnan, Mat. Sc. Eng. 32, 201 (1995).

[3] B.D. Hughes, Random walks and random environments, vol. 2, Clarendon Press, Oxford, 1996.

[4] J. Machta, Phys. Rev. B 24, 5260 (1981).

[5] C. Van den Broeck, Phys. Rev. Lett. 62, 1421 (1989).

[6] R. Burioni and D. Cassi, J. Phys. A 38, R45 (2005).

[7] J.J. Kozak, Adv. Chem. Phys. 115, 245 (2000).

[8] E.W. Montroll, J. Math. Phys 10, 753 (1969).

[9] B. Kahng and D. Redner, J. Phys. A 22, 887 (1989).

[10] O. Matan and S. Havlin, Phys. Rev. A 40, 6573 (1989).

[11] J.J. Kozak and V. Balakrishnan, Phys. Rev. E 65, 021105 (2002).

[12] S. Redner, A Guide to First-Passage Processes, Cambridge University Press, New York, 2001.

[13] A. Baronchelli and V. Loreto, Phys. Rev. E 73, 026103 (2006).

[14] C. Van den Broeck, Phys. Rev. A 40, 7334 (1989).

[15] S. Condamin, O. Bénichou, V. Tejedor, R. Voituriez and
J. Klafter, Nature 450, 77 (2007).

[16] E. Agliari, R. Burioni, D. Cassi and F.M. Neri, Theor. Chem. Acc. (2007).

[17] G. Oshanin, O. Bénichou and A. Blumen, J. Stat. Phys. 112, 541 (2003).

[18] G.H. Weiss, J. Stat. Phys. 24, 587 (1981).

[19] G. Oshanin, O. Bénichou, M. Coppey and M. Moreau, Phys. Rev. E 66, 060101(R) (2002).

[20] R. Burioni, D. Cassi and S. Regina, Physica A 265, 323 (1999).

[21] A. Maritan, G. Sartoni and A.L. Stella, Phys. Rev. Lett. 71, 1027 (1993).

[22] R. Burioni, D. Cassi, F. Corberi and A. Vezzani, Phys. Rev. E 75, 011113 (2007).

[23] M. Knežević and D. Knežević, Phys. Rev. E 53, 2130 (1996).

[24] P.G. de Gennes, J. Chem. Phys. 76, 3316 (1982).

[25] A. Blumen, G. Zumofen and J. Klafter, Phys. Rev. B 30, 5379 (1984). 